\title{
Simple analytical method for wireless distributed networks considering contending and hidden nodes
}

\author{
Hiraku Okada ${ }^{1 a)}$ and Kenichi Mase ${ }^{2}$ \\ ${ }^{1}$ EcoTopia Science Institute, Nagoya University \\ Furo-cho, Chikusa-ku, Nagoya 464-8603, Japan \\ ${ }^{2}$ Graduate School of Science and Technology, Niigata University \\ 8050 Ikarashi 2-no-cho, Nishi-ku, Niigata 950-2181, Japan \\ a)hiraku@m.ieice.org
}

\begin{abstract}
In this letter, we introduce a simple analytical model for wireless distributed networks (WDNs) using IEEE 802.11 distributed coordination function (DCF), which considers contending and hidden nodes. Furthermore, we derive simple formulas of throughput for maximum and saturated cases, and discuss the characteristics of the influence of the contending and hidden nodes. We also compare the analytical results with the simulation of 802.11 DCF. Although our analytical model simplifies the behavior of 802.11 DCF, analytical results coincide with the simulation.
\end{abstract}

Keywords: wireless distributed network, queueing model, exposed node problem, hidden node problem

Classification: Terrestrial Wireless Communication/Broadcasting Technologies

\section{References}

[1] ANSI/IEEE Std 802.11, Wireless LAN medium access control (MAC) and physical layer (PHY) specifications, 1999.

[2] G. Bianchi, "Performance analysis of the IEEE 802.11 distributed coordination function," IEEE J. Sel. Areas Commun., vol. 18, no. 3, pp. 535-547, March 2000.

[3] H. Wu, F. Zhu, Q. Zhang, and Z. Niu, "Analysis of IEEE 802.11 DCF with hidden terminals," IEEE Global Telecommunications Conference, 2006.

[4] O. Ekici and A. Yongacoglu, "IEEE 802.11a throughput performance with hidden nodes," IEEE Commun. Lett., vol. 12, no. 6, pp. 465-567, June 2008.

[5] L. Kleinrock, Queueing systems volume 1; Theory, John Wiley \& Sons, 1975.

[6] Scalable Network Technologies, [Online] http://www.scalable-networks.com/. 
[7] H. Okada and K. Mase, "Performance analysis of wireless mesh networks with three sector antennas," International Conference on Wireless Communications and Mobile Computing, pp. 1232-1236, July 2010.

\section{Introduction}

In wireless distributed networks (WDNs), a lot of nodes are deployed in an area, and they construct a wireless network by an autonomously distributed manner. WDNs have some useful applications such as wireless mesh networks and mobile ad hoc networks, and attract much attention for its flexibility and extensibility.

Most wireless mesh networks and mobile ad hoc networks employ IEEE 802.11 wireless LAN [1] as a wireless media. IEEE 802.11 distributed coordination function (DCF) is based on carrier sense multiple access with collision avoidance (CSMA/CA), which contains a backoff mechanism to avoid collisions. In addition, exposed and hidden node problems are serious in WDNs, and they complicate the characteristics of WDNs.

There are a lot of work about analysis of 802.11 DCF, and most of them are based on [2]. In [2], the author introduces a two-dimensional Markov chain to represent backoff behavior, and derives the throughput in the saturated situation. In $[3,4]$, the authors extend [2] so as to consider the influence of hidden nodes. These work can describe accurate behavior of 802.11 DCF, but the derived formula is complex and it is difficult to understand the characteristics easily from the formula.

In this letter, we introduce a simple analytical model and derives throughput for WDNs, which considers contending and hidden nodes. Furthermore, we derive simple formulas of throughput for maximum and saturated cases, and discuss the characteristics of the influence of the contending and hidden nodes. We carry out the simulation of 802.11 DCF and compare the analytical results with the simulation.

\section{Simple analytical model}

Figure 1 shows a network model for analysis. A sender node (tx) transmits a packet to a receiver node (rx). Beside the sender node, some contending nodes exist. Hidden nodes are also located around the receiver node. Let $m$ and $n$ be the numbers of contending and hidden nodes for the sender node,

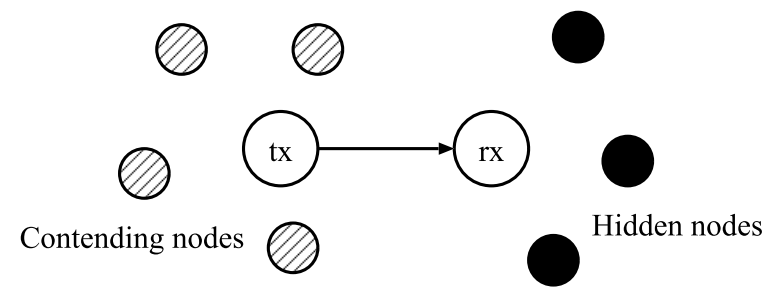

Fig. 1. A network model for analysis. 
respectively. When each node has multiple interfaces, it can use multiple channels. In this case, each node selects one of $s$ channels for transmission of a packet, where $s$ is the number of available channels.

Each node generates a packet so that a birth interval obeys exponential distribution with a birth rate $\lambda$. Fixed packet length is assumed, and it is denoted by $L_{\mathrm{p}}$ bits. The time duration of a packet is $T_{\mathrm{p}}=L_{\mathrm{p}} / R$, where $R$ bits/s is a transmission rate. An offered load is defined as a traffic intensity of generating packets, and that per node is expressed by $g=\lambda T_{\mathrm{p}}$. This letter considers two buffer models. One is an infinite buffer model, where each node has an infinite buffer. The other is a non-buffer model, that is, each node does not have a buffer except for waiting packet transmission and retransmission.

Let us assume ideal CSMA, in which packets sent by contending nodes can avoid collisions and they are processed as first-in first-out manner. Indeed, this assumption is ideal, but it is enough to investigate the influence of contending nodes. Note that packets sent by hidden nodes cause collisions. Under the ideal CSMA assumption, one-hop transmission among contending nodes can be modeled as an $\mathrm{M} / \mathrm{D} / s / / m^{\prime}$ queue for the infinite buffer model and as an $\mathrm{M} / \mathrm{D} / \mathrm{s} / \mathrm{s} / \mathrm{m}^{\prime}$ queue for the non-buffer model, where $m^{\prime}$ is the number of contending nodes including the sender node, and can be expressed by $m^{\prime}=m+1$. This letter employs these simple queueing models for throughput analysis.

\section{Analysis}

\subsection{Channel load}

An effective load is defined as traffic intensity of newly generating and retransmitting packets. The effective load per node can be expressed by $g_{\mathrm{e}}=\mathrm{Ng}$, where $N$ is the average number of transmissions until a packet is transmitted successfully.

Let a channel load be average traffic intensity of on-transmitting packets on all available channels for all nodes. For the infinite buffer model, the channel load can be expressed by

$$
G_{\mathrm{c}}= \begin{cases}m^{\prime} g_{\mathrm{e}} & \left(m^{\prime} g_{\mathrm{e}}<s\right) \\ s & \left(m^{\prime} g_{\mathrm{e}} \geq s\right)\end{cases}
$$

because any packet losses happen in $\mathrm{M} / \mathrm{D} / \mathrm{s} / / \mathrm{m}^{\prime}$ queueing model. For the non-buffer model, a steady state occupation probability, which is the probability that the number of buffering packets is $k$, of the $\mathrm{M} / \mathrm{D} / \mathrm{s} / \mathrm{s} / \mathrm{m}^{\prime}$ queueing model may be derived by [5]

$$
\pi_{k}=\frac{\left(\begin{array}{c}
m^{\prime} \\
k
\end{array}\right) g_{\mathrm{e}}^{k}}{\sum_{i=0}^{s}\left(\begin{array}{c}
m^{\prime} \\
i
\end{array}\right) g_{\mathrm{e}}^{i}} .
$$

From this probability, the channel load can be obtained by

$$
G_{\mathrm{C}}=\sum_{k=0}^{s} k \pi_{k}
$$


For a single channel case $(s=1),(3)$ can be simply expressed by

$$
G_{\mathrm{c}}=\frac{m^{\prime} g_{\mathrm{e}}}{1+m^{\prime} g_{\mathrm{e}}} .
$$

\subsection{Throughput}

Let us derive a packet transmission successful probability, $Q_{\mathrm{s}}$, from the channel load. In this letter, the cause of transmission failure of a packet is collision only with packets from hidden nodes. The hidden nodes send packets in spite of channel state (busy or idle) at the sender node. Like a pure ALOHA, a packet is transmitted successfully if the hidden nodes do not send any packets during twice of a packet duration. The channel load per channel per node is $G_{\mathrm{c}} / s m^{\prime}$. Considering the number of hidden nodes, the packet transmission successful probability may be expressed by

$$
Q_{\mathrm{s}}=\left\{e^{-2 G_{\mathrm{c}} / s m^{\prime}}\right\}^{n}=e^{-2 n G_{\mathrm{c}} / s m^{\prime}},
$$

for $n \neq 0$. Obviously, $Q_{\mathrm{s}}=1$ for $n=0$ (no hidden nodes). The average number of transmissions can be obtained by $N=1 / Q_{\mathrm{s}}$.

Throughput is defined as traffic intensity of successfully transmitted packets. The throughput per node can be derived by multiplying the channel load per node and the packet transmission successful probability, that is,

$$
\eta=\frac{G_{\mathrm{c}}}{m^{\prime}} Q_{\mathrm{s}}=\left\{\begin{array}{ll}
\frac{G_{\mathrm{c}}}{m^{\prime}} e^{-2 n G_{\mathrm{c}} / s m^{\prime}} & (n \neq 0) \\
\frac{G_{\mathrm{c}}}{m^{\prime}} & (n=0)
\end{array} .\right.
$$

Let us consider two specific cases. One is a saturated situation, in which the offered load comes close to infinite value. The other is a maximum throughput case. In the saturated situation, the channel is always busy because of the ideal CSMA. Then, the channel load is equal to the number of available channels. By substituting $s$ for $G_{\mathrm{C}}$ in (6), the throughput per node in the saturated situation is derived by

$$
\hat{\eta}=\left\{\begin{array}{ll}
\frac{s}{m^{\prime}} e^{-2 n / m^{\prime}} & (n \neq 0) \\
\frac{s}{m^{\prime}} & (n=0)
\end{array} .\right.
$$

From the derived function of (6), the maximum throughput per node can be obtained. When $n \neq 0$, the throughput per node is maximized at $G_{\mathrm{c}}=$ $s m^{\prime} / 2 n$. For $n=0$, the maximum throughput per node is obtained when $G_{\mathrm{C}}$ comes closed to infinity, that is, the saturated situation. Then, the maximum throughput per node may be expressed by

$$
\eta_{\max }=\left\{\begin{array}{ll}
\frac{s}{2 n} e^{-1} & (n \neq 0) \\
\frac{s}{m^{\prime}} & (n=0)
\end{array} .\right.
$$


Table I. Simulation settings.

\begin{tabular}{|l|l|}
\hline simulator & QualNet 4.5.1 $[6]$ \\
\hline simulation duration & $180 \mathrm{~s}$ \\
\hline node allocation & $\begin{array}{l}\text { tx and rx only }(m=0, n=0) \\
\text { with contending nodes }(m=2, n=0) \\
\text { with hidden nodes }(m=0, n=2)\end{array}$ \\
\hline MAC/PHY & IEEE 802.11b (transmission rate: 2 Mbps) \\
\hline buffer size & 50 kbytes \\
\hline application flow & $\begin{array}{l}\text { UDP datagram } \\
\text { (generation interval: exponential, length: } 1024 \text { bytes) }\end{array}$ \\
\hline
\end{tabular}

\subsection{Discussion}

From the analytical results of two specific cases (7), (8), the influence of contending and hidden nodes is discussed.

When hidden nodes exist, the throughput of the saturated situation increases as the number of contending nodes increases. This reason is that the increase in the number of contending nodes reduces the packet transmissions from the hidden nodes and it results in the reduction of the collision probability. On the other hand, the throughput of the saturated and the maximum cases is exponentially degraded as the number of hidden nodes increases. It is confirmed that the hidden node problem is much more serious than the exposed node problem. On the other hand, the throughput is inversely degraded by the number of contending nodes for the case of no hidden nodes. In this case, any collisions do not occur, and contending nodes share the available channels.

\section{Numerical results}

Simulation settings are shown in Table I. QualNet [6] is employed as a network simulator. IEEE $802.11 \mathrm{~b}$ with $2 \mathrm{Mbps}$ is used as a media access control (MAC) and physical (PHY) system. We consider 3 node allocations: tx and rx only $(m=0, n=0)$, with contending nodes $(m=2, n=0)$, and with hidden nodes $(m=0, n=2)$. In all allocations, the distances between the sender node and the receiver node is $450 \mathrm{~m}$, which is within the communication range. For the case with contending nodes, the contending nodes are located at $450 \mathrm{~m}$ of right-hand side of the receiver node, seeing from the sender node. The distance between the sender and contending nodes is about $636 \mathrm{~m}$, which is within the carrier sense range. For the case with hidden nodes, the hidden nodes are located at $450 \mathrm{~m}$ on the opposite side of the receiver node from the the sender node. The distance between the sender node and the hidden node is $900 \mathrm{~m}$, which is beyond the carrier sense range. The buffer size of each node is 50 kbytes. An application flow uses UDP datagram. Its generation interval obeys the exponential distribution and its length is 1024 bytes. The birth rate is adjusted according to the offered load.

Figure 2 shows the throughput per node. Analytical results of both the infinite buffer and the non-buffer models are shown. Simulation results are 


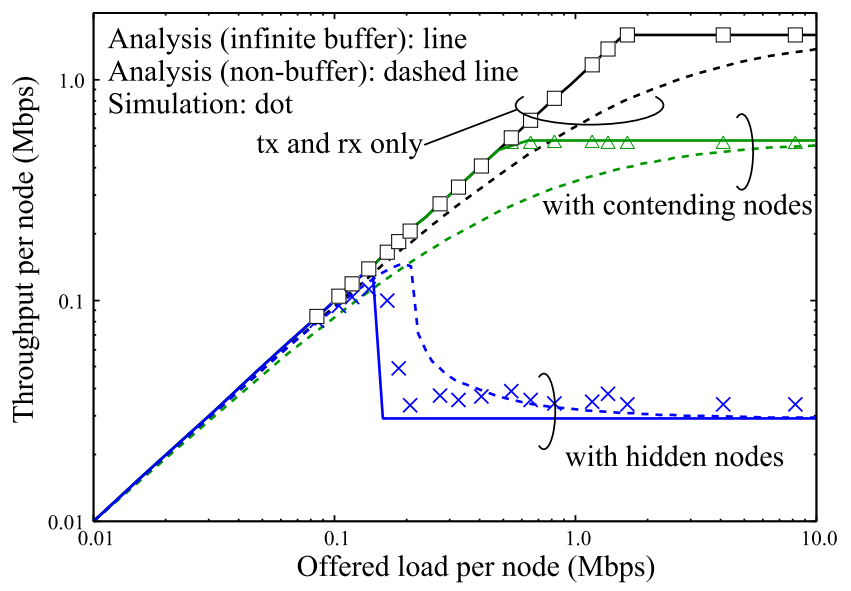

Fig. 2. Throughput performance considering contending and hidden nodes.

also depicted with dots. The analytical values of both the offered load and the throughput are multiplied by the maximum throughput for the case of tx and $\mathrm{rx}$ only in the simulation, where the maximum throughput is about $1.59 \mathrm{Mbps}$. It is confirmed that the analytical results of the infinite buffer coincide with the simulation results when any hidden nodes exist. For the case with hidden nodes, packet collisions often happen due to hidden nodes, and the number of retransmissions increases. Some packets will go over a retry limit, and will be lost. Therefore, simulation results are between the analytical results of the infinite buffer and the non-buffer models although they are well-matched with the analytical results. From Fig. 2, it is also conducted that the analytical results of the maximum and saturated throughput are very similar to the simulation results of those.

\section{Conclusions}

In this letter, we have proposed the simple analytical model for WDNs, and compared the analytical results with the simulation. Although our analytical model simplifies the behavior of 802.11 DCF, analytical results coincide with the simulation. Therefore, our analytical model is very useful to investigate the influence of contending and hidden nodes.

In the proposed method, the main points of simplification are ignoring packet collisions from contending nodes, ignoring the effect of contention window, and independent behavior of contending and hidden nodes with each other. These assumptions may not be satisfied if the number of contending and hidden nodes is very large. However, the proposed method is effective as far as the characteristics of the system can be estimated. In addition, our analytical method can be used for not only simple WDNs but also some extension of WDNs, e.g. [7].

\section{Acknowledgments}

This work was supported by JSPS KAKENHI. 